\title{
Evaluation of the immune state activation in patients affected by $\mathrm{ONJ}$ : preliminary data
}

\author{
Ilaria Roato ${ }^{1}$, Chiara Motta ${ }^{1}$, Federico Mussano ${ }^{1}$, Alessio Gambino ${ }^{1}$, Francesco Maria Erovigni ${ }^{1}$ \\ 1 University of Turin
}

Funding: The author(s) received no specific funding for this work.

Potential competing interests: The author(s) declared that no potential competing interests exist.

\section{Abstract}

Bisphosphonate (BP)-associated osteonecrosis of the jaw (ONJ) is a serious adverse event characterized by nonhealing necrotic bone tissue of mandible or maxilla. BPs target osteoclasts, inhibiting their action and blocking bone resorption, nonetheless an increased bone resorption in the oral cavity is associated to ONJ. One of the causes of ONJ is the dysregulation of the immune system, in particular the strong reduction of gamma/delta T cells circulating in peripheral blood (PB). The rate of circulating osteoclast precursors (OCPs) is altered in bone metastatic patients, suggesting a systemic alteration of osteoclast compartment, but we do not know wether it is altered in cancer patients affected by active lesion of ONJ induced by Zoledronic acid treatment. By flow cytometric analysis of patients' and controls' PB cells, we studied the presence of different T cell subsets, according to the expression of gamma/delta chains, CD4, CD8, CD25 and CD69 and of OCPs. We also evaluated the capability of PBMCs to spontaneously differentiate into osteoclasts in vitro, which is an index of pathological bone resorption. Our preliminary results confirmed in ONJ patients a marked reduction in gamma/delta T cells compared to their level befor patients started BP treatment. The subsets of activated T cells and OCPs were not significantly modified. In ONJ patients, in vitro osteoclastogenesis was comparable to the one of healthy control, while before patients started BP treatment osteoclastogenesis was significantly increased. This result suggests that BPs correctly act in reducing the systemic activation of osteoclasts, associated to bone metastatic patients, as expected, but BPs fail to block osteoclast activity locally.

Background: Bisphosphonate (BP)-associated osteonecrosis of the jaw (ONJ) is a serious adverse event characterized by non-healing necrotic bone tissue of mandible or maxilla. BPs target osteoclasts, inhibiting their action and blocking bone resorption, nonetheless an increased bone resorption in the oral cavity is associated to ONJ. One of the causes of ONJ is the dysregulation of the immune system, in particular the strong reduction of gamma/delta T cells circulating in peripheral blood (PB). The rate of circulating osteoclast precursors (OCPs) is altered in bone metastatic patients, suggesting a systemic alteration of osteoclast compartment.

Materials and Methods: PB cells derived from patients with bone metastases before BP treatment, patients with BPinduced ONJ and controls were studied for the presence of different T cell subsets, according to the expression of gamma/delta chains, CD4, CD8, CD25 and CD69 and of OCPs, by flow cytometric analysis. We also evaluated the 
capability of PBMCs to spontaneously differentiate into osteoclasts in vitro, which is an index of an active pathological bone resorption.

Results: Our preliminary data confirmed a marked reduction in gamma/delta T cells in ONJ patients, according to literature data, while the subsets of activated T cells and OCPs were not significantly modified. In patients with bone metastases, before starting of BPs, the level of gamma/delta T cells was normal. In ONJ patients, in vitro osteoclastogenesis was comparable to the one of healthy control, while before patients started BP treatment, osteoclastogenesis was significantly increased compared to the control.

Conclusions: These preliminary results confirmed the previously described reduction of circulating gamma/delta T cells in ONJ patients and suggest the possibility to monitor the level of gamma/delta T cells during the course of the BP treatment as predictive marker of ONJ development. Moreover, we observed that BPs correctly act in reducing the systemic activation of osteoclasts, associated to bone metastatic patients, as expected, since spontaneous osteoclastogenesis was not present. Nonetheless, BPs fail to block osteoclast activity locally, in the oral cavity. 